Annales Geophysicae (2001) 19: 1399-1409 (C) European Geophysical Society 2001

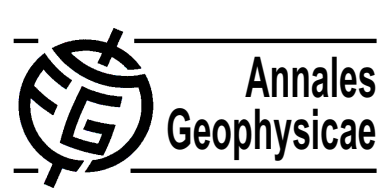

\title{
Cluster magnetic field observations of the bowshock: Orientation, motion and structure
}

\author{
T. S. Horbury ${ }^{1}$, P. J. Cargill ${ }^{1}$, E. A. Lucek ${ }^{1}$, A. Balogh ${ }^{1}$, M. W. Dunlop ${ }^{1}$, T. M. Oddy ${ }^{1}$, C. Carr ${ }^{1}$, P. Brown ${ }^{1}$, A. Szabo ${ }^{2}$, \\ and K.-H. Fornaçon ${ }^{3}$ \\ ${ }^{1}$ The Blackett Laboratory, Imperial College, London, UK \\ ${ }^{2}$ Goddard Space Flight Center, Greenbelt, MD, USA \\ ${ }^{3}$ Technische Universität Braunschweig, Braunschweig, Germany
}

Received: 21 March 2001 - Revised: 18 June 2001 - Accepted: 19 June 2001

\begin{abstract}
Four spacecraft Cluster magnetic field observations of the low $\beta$ quasi-perpendicular terrestrial bowshock are presented for the first time. Multiple quasi-perpendicular crossings on 25 December 2000 are analysed. By combining data from the four spacecraft, bowshock orientations and velocities can be calculated. It is shown that, even while in rapid motion, the bowshock normal direction remains remarkably constant, and that coplanarity estimates are accurate to, typically, around $20^{\circ}$. Magnetic field magnitude profiles are shown to be very well correlated between spacecraft although downstream waves with fluctuations perpendicular to the local field, while statistically similar at all four spacecraft, are poorly correlated on separation scales of several hundred $\mathrm{km}$. Examples are shown of a number of bowshock phenomena, including non-standing fluctuations in the shock foot and the shock interacting with changing solar wind conditions.
\end{abstract}

Key words. Interplanetary physics (planetary bow shocks) Space plasma physics (shock waves; waves and instabilities)

\section{Introduction}

Our knowledge of the bow shock currently stems largely from data obtained by dual spacecraft measurements (the ISEE-1 and -2 and AMPTE-IRM and -UKS missions). Broadly speaking, the bow shock can be split up into two regimes, depending on the angle between the shock normal and the interplanetary magnetic field (usually $\theta_{\mathrm{BN}}$ ). When $\theta_{\mathrm{BN}}<(>) 45^{\circ}$, the shock is said to be quasi-parallel (quasi-perpendicular) (Schwartz et al., 1983). In the former case (see, for example, the review of Krauss-Varban, 1995), shock-heated ions can escape upstream, giving rise to a very extended shock structure. The quasi-perpendicular shock is believed to be more compact (e.g. Goodrich, 1985; Scudder et al., 1986) and is the focus of this study.

Correspondence to: T. S. Horbury (t.horbury@ic.ac.uk)
Collisionless shock structure is dominated by ion dynamics in all regimes encountered at the bow shock. Through data analysis (Sckopke et al., 1983) and numerical simulations (Leroy et al., 1982) of quasi-perpendicular shocks, it was established that a small fraction (20\%) of the solar wind ions are reflected by an electric potential at the shock front. These reflected ions then form a ring/beam distribution function, which is unstable to an electromagnetic Alfvén cyclotron instability. The non-linear evolution of this instability then leads to thermalisation of the unstable distribution with strong heating of the ions. Thus the energy flow at the shock is from solar wind kinetic energy, into the ring/beam distribution and finally into plasma heating. It was believed in most regimes that the reflection process was steady, although for high Alfvén Mach number (Quest, 1986) and low ion$\beta$ (Goodrich and Cargill, 1986, unpublished results) there is evidence that this is not the case.

In this paper we will be primarily concerned with magnetic field observations. Parallel to the shock normal, the above considerations lead to a magnetic field profile that first increases slowly above the solar wind values (the shock foot), and then more abruptly (the shock ramp where the ion reflection occurs). The width of the foot is roughly $V_{\mathrm{SW}} / \Omega_{\mathrm{i}}$, where $V_{\mathrm{SW}}$ is the solar wind speed and $\Omega_{\mathrm{i}}$ the upstream ion cyclotron frequency. For typical values $\left(V_{S W}=400 \mathrm{~km} / \mathrm{s}\right.$ and $\Omega_{\mathrm{i}}=1 \mathrm{rad} / \mathrm{s}$ ), this distance is a few hundred $\mathrm{km}$. The true scaling of the ramp is much less clear (e.g. Scudder et al., 1986) but something on the order of $30-50 \mathrm{~km}$ is reasonable. The magnetic field in the ramp eventually peaks at an overshoot, at least for high Mach number (supercritical) shocks, subsequently relaxing to the downstream value predicted by the Rankine-Hugoniot conditions. This picture seems to be confirmed by spacecraft data and onedimensional numerical simulations. In addition, for low Mach number (subcritical) shocks, there is the possibility of upstream whistler (right-handed) waves. However, the magnetic field structure along the shock front is less clear. The twin spacecraft data from the 1970s and 1980s generally had one spacecraft following the other along the shock normal 


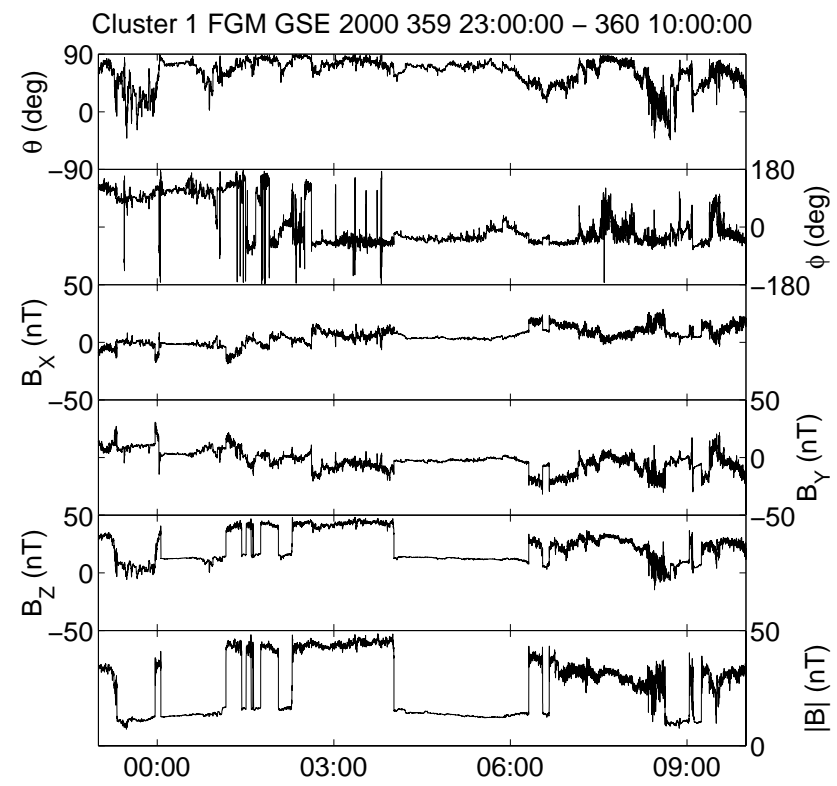

Fig. 1. The magnetic field in GSE coordinates at Cluster 1 from 23:00 on 24 December 2000 until 10:00 on 25 December.

and so could not shed light on this question. Numerical simulations using two and three dimensional hybrid simulations (massless fluid electrons and kinetic ions: Winske and Quest, 1988; Thomas, 1989; McKean et al., 1995) have provided some clues. Winske and Quest (1988) showed that the reflected ions were indeed thermalised by an Alfvén ion cyclotron instability propagating obliquely with respect to the magnetic field. Later work (McKean et al., 1995) also identified mirror mode fluctuations. Thus one would expect a shock structure that had "ripples" along the magnetic field, i.e. along the shock front. For the cases examined by Winske and Quest (an Alfvén Mach number of 8), the wavelength of the field fluctuations along the shock appeared to be a few times the upstream ion inertial length, $c / \omega_{\mathrm{i}}$, typically around $200 \mathrm{~km}$. A study of the field structures showed that the field amplitude varied by a factor of 2-3 in the direction along the shock. These results were generally confirmed by the (rather spatially limited) 3-D hybrid simulations of Thomas (1989). In addition, the instability resulted in a very rapid thermalisation of the reflected ions; the main ion anisotropy with respect to the magnetic field is removed within the shock ramp (Winske and Quest, 1988).

Observations (Lacombe et al., 1992; Russell and Farris, 1995; Hubert et al., 1989) reveal the presence of both Alfvén ion cyclotron and mirror modes downstream of quasiperpendicular shocks, in general agreement with simulations (e.g. McKean et al., 1995).

The Cluster mission is ideally suited to investigating the multi-dimensional structure of the perpendicular bow shock. The spacecraft configuration permits investigations of many shock phenomena, such as the steadiness of the shock structure; the structure along the shock surface; waves accompanying the shock; and shock response to changing upstream conditions.
In this paper, we concentrate on magnetic field measurements made by the fluxgate magnetometer (Balogh et al., 2001) of a set of bowshock crossings on 25 December 2000, when the solar wind plasma $\beta$ was unusually low (around 0.1 ), the Alfvén Mach number was around 4-6, and the magnetic field pointed nearly due northward during much of the day; as a result, the shocks were generally quasiperpendicular. The following section provides an overview of the day, estimates of bowshock normals using four spacecraft timings and comparisons with coplanarity and model calculations. A "clean," supercritical, quasi-perpendicular shock is then discussed in detail, with emphasis on interspacecraft comparisons of fluctuations. Finally, a number of other shock crossings are described briefly, illustrating the wide variety of phenomena visible: non-stationary fluctuations in the shock foot; the bowshock encountering solar wind discontinuities; magnetosheath response to bowshock motion; and quasi-periodic bowshock motion.

\section{December 2000: overview}

The four Cluster spacecraft left the magnetosheath and passed into the solar wind late on 24 December, outbound towards apogee. They encountered the bowshock several (around 20) times over the next 10 hours before re-entering the sheath. Magnetic field data taken between 23:00 on 24 December and 10:00 on 25 December by spacecraft 1 are shown in Fig. 1. During this time, the spacecraft were typically several hundred km apart and, on the scale of Fig. 1, their time series are very similar. At 00:00 on 25 December, spacecraft 1 was at a GSE $(X, Y, Z)$ position of $(7.3,18.2$, 1.7) $R_{E}$ and at $(8.2,15.1,-3.3)$ at $09: 00$. The formation was moving slowly in the $-Y$ and $-Z$ directions during this time, on the dusk flank at around 16:00 local time.

It can be seen from Fig. 1 that the upstream interplanetary magnetic field (IMF) was around 10-15 nT throughout this period. Unusually, the field pointed nearly northward $\left(\theta \approx 90^{\circ}\right)$ for most of the interval. As a result, the shocks encountered by the Cluster formation at this time were quasiperpendicular. There was a period between 08:30 and 09:00 when the field direction was closer to radial, however; we return to this interval later in this paper.

The Cluster spacecraft were still undergoing commissioning at this time and plasma instruments were switched off on all four spacecraft. However, plasma conditions at the L1 libration point measured by ACE are likely to have been similar to those immediately upstream of the Earth during this day. Measurements from ACE show a proton number density of around $10-30 \mathrm{~cm}^{-3}$, a proton temperature around $3 \times 10^{4} \mathrm{~K}$ and a solar wind velocity of about $350 \mathrm{~km} / \mathrm{s}$. As we show later, it is possible to estimate the upstream solar wind speed from the relative timings of solar wind structures at the four Cluster spacecraft and this procedure results in estimates similar to those at ACE.

It is possible to calculate the orientation and velocity of a plane using four spacecraft data (e.g. Song and Russell, 
2000). In essence, if one can identify four times at which a structure passes the four spacecraft and if one knows the location of the four spacecraft at those times, the normal $\boldsymbol{n}$ of the plane and its velocity along the normal, $\boldsymbol{V} \cdot \boldsymbol{n}$, can be calculated. This procedure assumes that the shock is approximately planar over the separation scale of the spacecraft (around $1000 \mathrm{~km}$ here) and is not accelerating significantly over the time between the observations (up to 1 minute but typically a few seconds). Considerably more sophisticated orientation estimation methods (e.g. Dunlop and Woodward, 1998; Dunlop et al., 2001) can be used to derive curvature and acceleration of the structures but these are not necessary for our purposes here.

It is not possible to estimate the velocity of a planar structure perpendicular to its normal using relative timings. That is, one can estimate the projection of the structure's velocity along the normal but not that perpendicular to it. To estimate its full vector velocity, it is necessary to assume a direction of motion. For the Earth's bowshock, this direction could be in the GSE $+X$ direction, for example. However, in this work we assume that it is parallel to the calculated normal. Of the shocks observed during 25 December, 16 could be identified accurately at all four spacecraft. Normals and velocities of these events calculated from inter-spacecraft timings are presented in Table 1. It is immediately apparent from the Table that the bowshock normal is rather constant, even though its velocity can vary from under $3 \mathrm{~km} / \mathrm{s}$ to over $70 \mathrm{~km} / \mathrm{s}$. This is clearer in Fig. 2, which shows timing-derived normals (black vectors) at their observed locations projected on the $X-Y$ and $X-Z$ GSE planes. The normals are generally consistent between themselves and a gradual change in normal direction can be observed along the Cluster orbit. Therefore, even while the bowshock is in rapid motion as a result of changing solar wind conditions, its normal remains remarkably constant. This consistency between normal estimates suggests that timing and positional uncertainties in the data are not sufficient to introduce significant errors in the normal estimates and, therefore, normals can be reliably estimated using spacecraft separated by several hundred $\mathrm{km}$. In addition, the assumptions of planarity and low acceleration must also be approximately satisfied on these scales for the timing derived normals to be in such close agreement.

It is of interest to compare normals calculated from interspacecraft timings with those derived from other methods. Model normals were calculated for 3 times during 25 December, using the Peredo et al. (1995) model and observed solar wind parameters from the ACE SWEPAM instrument. This model is parameterised by solar wind ram pressure, Alfvén and slow mode Mach numbers and interplanetary magnetic field (IMF) clock angle. The model normals are shown in Fig. 2 as long blue lines. It is clear that, while the predicted bowshock position is somewhat Earthward of that observed, the normal agrees extremely well with that calculated from the inter-spacecraft timings.

The coplanarity unit vector, $\left(\boldsymbol{B}_{u} \times \boldsymbol{B}_{d}\right) \times\left(\boldsymbol{B}_{u}-\boldsymbol{B}_{d}\right) / /\left(\mathbf{B}_{u} \times\right.$ $\left.\mathbf{B}_{d}\right) \times\left(\mathbf{B}_{u}-\mathbf{B}_{d}\right) \mid$, was also calculated for the shocks in Fig. 2 . This direction is expected to lie parallel to the shock normal
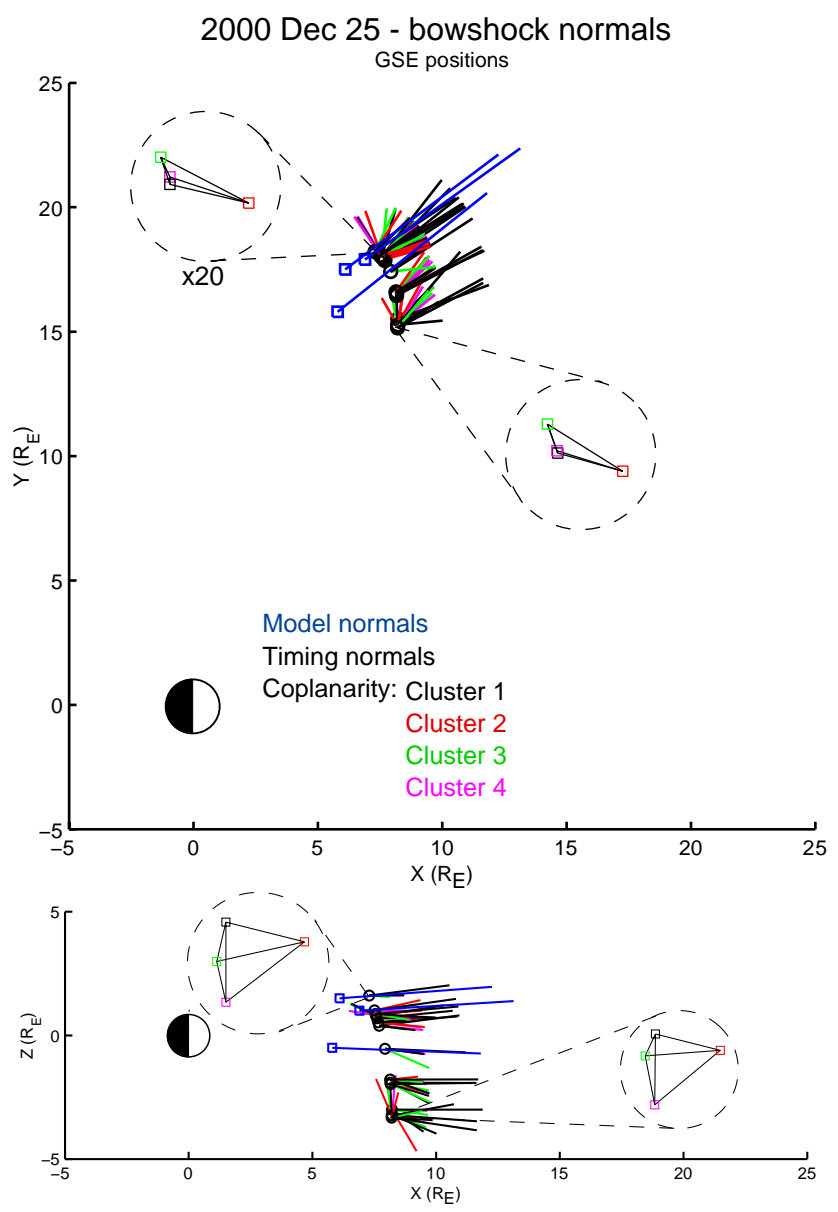

Fig. 2. Bowshock normals calculated from four spacecraft timings. Normals are plotted as long black lines extending from the observed bowshock locations, in (top) the $X-Y$ and (bottom) $X-Z$ planes. Shorter coloured lines show coplanarity normals calculated using magnetic field data from all four spacecraft. Long blue lines show calculated model normals. Relative spacecraft positions are shown for the start and end of the period of interest, expanded 20 times larger than reality.

on the basis of the MHD Rankine-Hugoniot relations. The up and downstream magnetic field vectors, $\boldsymbol{B}_{u}$ and $\boldsymbol{B}_{d}$, were calculated from the averages of $500 \mathrm{~km}$ of field data (where distances were calculated using the observed shock velocity), taken $500 \mathrm{~km}$ up and downstream of each shock. Coplanarity vectors are shown in Fig. 2 as short coloured lines (black, red, green and magenta for spacecraft 1,2, 3 and 4, respectively; the same colouring scheme is used throughout this paper to identify the four spacecraft). Agreement between coplanarity vectors and timing-derived normals is sometimes good but can also be very poor; the mean angle between the coplanarity vectors and the timing-derived normals is $30 \pm 6^{\circ}$ and the median is $23^{\circ}$. This discrepancy is not a strong function of the distance over which coplanarity vectors are calculated; for $1000 \mathrm{~km}$ up and downstream, separated by $1000 \mathrm{~km}$ from the shock, the mean angle was $36 \pm 6^{\circ}$ while, for $100 \mathrm{~km}$, it was $25 \pm 6^{\circ}$. In particular, there appears, from Fig. 2, to 
Table 1. Bowshock crossings on 25 December 2000. Times listed are for spacecraft 1 . Normal unit vector estimates in GSE coordinates are shown as well as velocities of the shocks, where negative velocities correspond to inward bowshock motion and vice versa

\begin{tabular}{crrrr}
\hline Time & Normal $X$ & Normal $Y$ & Normal $Z$ & $V(\mathrm{~km} / \mathrm{s})$ \\
\hline 00:03:41 & 0.827 & 0.553 & 0.105 & -26.2 \\
$01: 10: 07$ & 0.841 & 0.528 & 0.119 & 40.4 \\
$01: 26: 07$ & 0.854 & 0.514 & 0.076 & -48.3 \\
$01: 30: 39$ & 0.615 & 0.788 & -0.025 & 57.2 \\
$01: 35: 40$ & 0.700 & 0.706 & 0.112 & -11.3 \\
$01: 45: 27$ & 0.780 & 0.621 & 0.077 & 40.1 \\
$02: 03: 29$ & 0.818 & 0.573 & 0.061 & -26.1 \\
$02: 17: 41$ & 0.827 & 0.552 & 0.107 & 2.75 \\
$04: 01: 15$ & 0.838 & 0.544 & -0.037 & -35.3 \\
$06: 18: 43$ & 0.907 & 0.421 & 0.002 & 12.4 \\
$06: 32: 47$ & 0.873 & 0.487 & -0.005 & -9.5 \\
$06: 39: 32$ & 0.885 & 0.465 & 0.013 & 17.4 \\
$08: 37: 31$ & 0.937 & 0.351 & 0.001 & -7.6 \\
$09: 02: 22$ & 0.877 & 0.476 & -0.064 & 9.8 \\
$09: 05: 41$ & 0.641 & 0.758 & 0.119 & -72.7 \\
$09: 14: 41$ & 0.878 & 0.460 & -0.130 & 13.7 \\
\hline
\end{tabular}

be a systematic shift in coplanarity vectors in the $+Y$ direction from the timing-derived normal; this may be due to the presence of waves around the shocks. Detailed comparisons of shock normal estimators with inter-spacecraft timings will be discussed in a later paper but we note that, on the basis of the data in Fig. 2, coplanarity vectors appear to be accurate to within only around $20-30^{\circ}$.

Figure 2 also presents the relative positions of the four spacecraft within the formation at the beginning and end of the interval, expanded by a factor of 20 to make their orientations visible. Two key properties of the formation are notable. Spacecraft 1, 3 and 4 were in a plane nearly perpendicular to the GSE $X$ direction and hence nearly perpendicular to the solar wind flow; spacecraft 2 was around $1000 \mathrm{~km}$ upstream. As a result, spacecraft 2 observed Earthward propagating structures earlier than the other three spacecraft and vice versa. The formation shape did not change significantly during the day.

We now proceed to discuss several individual shocks in more detail, starting with a sharp quasi-perpendicular case.

\section{A clean quasi-perpendicular shock}

The first shock encountered on 25 December proved to be a clean one compared to those later in the day. Magnetic field data from all four spacecraft are shown in Fig. 3 at their times of observation. The bowshock was moving Earthward, and the four spacecraft passed from the magnetosheath into the solar wind as a result of its motion. Spacecraft 2 encountered the shock around 30 seconds before the remaining three

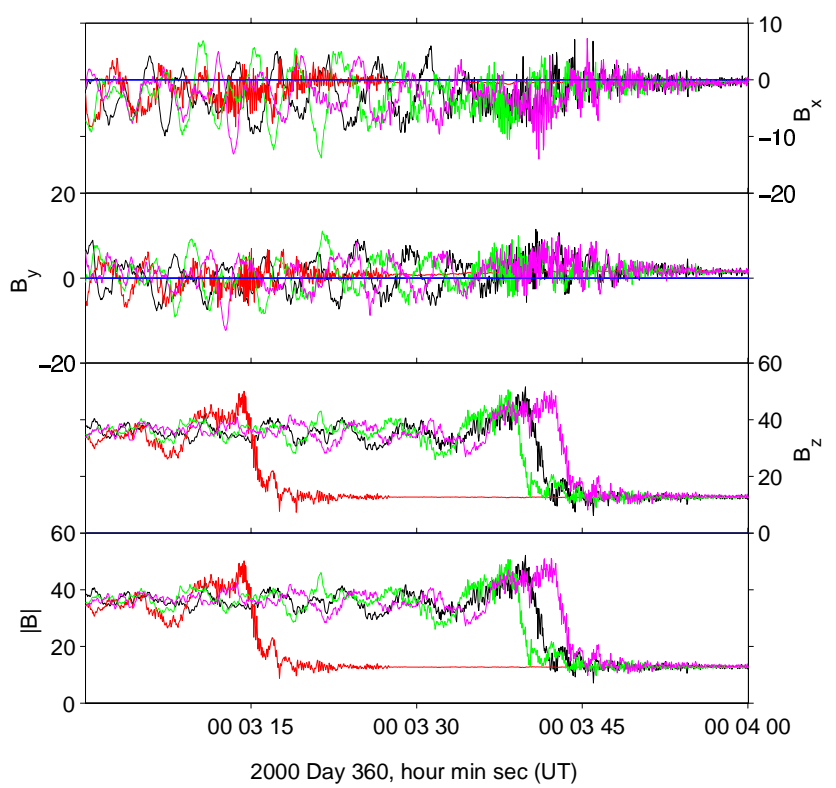

Fig. 3. High resolution (22 vectors/s) GSE magnetic field at all four Cluster spacecraft during a quasi-perpendicular bowshock crossing. Traces for each spacecraft are shown in a different colour: black, red, green and magenta for spacecraft 1,2, 3 and 4, respectively.

spacecraft, since it was significantly upstream of the others, as discussed in the previous section. Variations in $B_{Z}$ were very similar to those in the magnitude, since the field was almost directly Northward during this time.

The magnetic field magnitude profiles at the four spacecraft are rather similar, although downstream wave activity makes it difficult to distinguish them sensibly from Fig. 3. It is even harder to compare variations in the $B_{X}$ and $B_{Y}$ components, where large amplitude waves are clear in all four spacecraft time series.

This shock had a normal close to those of most of the other shocks in the day and a velocity of $26 \mathrm{~km} / \mathrm{s}$, also typical (see Table 1). The angle of the upstream magnetic field to the normal, $\theta_{\mathrm{BN}}$, was $87,82,86$ and 86 degrees at spacecraft $1,2,3$ and 4 , respectively, making this a nearly perpendicular shock. The plasma $\beta$ was very low, around 0.1. The Alfvén speed was around $67 \mathrm{~km} / \mathrm{s}$, making the Mach number around 4.8 .

With knowledge of the normal and the velocity, it is possible to transform spacecraft data from a time series into a spatial sample through the structure. To examine the consistency of the shock structure between spacecraft, all four data sets at the highest resolution ( 22 vectors/s) were converted into a distance perpendicular to the shock surface (i.e. parallel to its normal) using the calculated shock velocity; these spatial series are plotted together in Fig. 4. The data are plotted in a coordinate system with $B_{N}$ parallel to the shock normal, $B_{\perp 1}$ perpendicular to the normal and the mean field direction, and $B_{\perp 2}$ completing the right-handed set. Because $\theta_{\mathrm{BN}} \approx 90^{\circ}$, almost all the field was in the $B_{\perp 2}$ direction. This coordinate system is similar to the familiar $L M N$ system; in both cases, $N$ is parallel to the shock normal. The maximum variance 


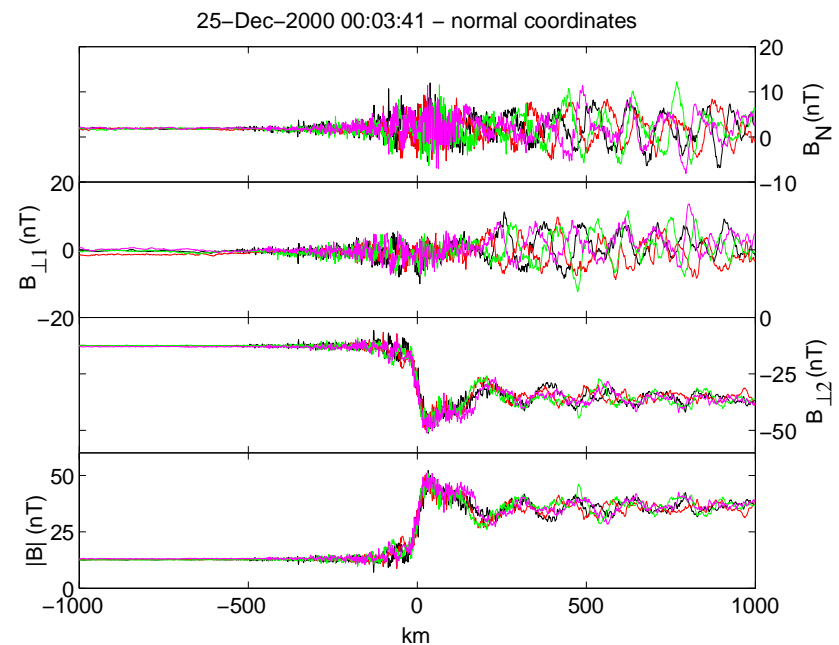

Fig. 4. High resolution magnetic field at all four Cluster spacecraft, during the quasi-perpendicular bowshock crossing shown in Fig. 3, plotted in terms of distance from the bowshock. Components are plotted in a coordinate system where $B_{N}$ is parallel to the shock normal, $B_{\perp 1}$ is perpendicular to the normal and the upstream field direction, and $B_{\perp 2}$ completes the right-handed set.

$(L)$ direction is, for the quasi-perpendicular shocks presented here, close to the mean field, $\perp 2$ vector, and therefore $\perp 1$ is close to the $M$ direction.

The consistency, in Fig. 4, between the field magnitude profiles at the four spacecraft is remarkable, with a similar ramp, overshoot, undershoot and downstream oscillation. These data demonstrate that the field magnitude profile through the shock was time-stationary with respect to the shock - that is, it was a standing oscillation, as previously shown in dual spacecraft observations (e.g. Scudder et al., 1986). There was some compressive wave activity in the foot of the shock (we return to upstream waves in later sections) as well as high frequency wave activity in all three components and the magnitude in the immediate vicinity $( \pm \approx 200 \mathrm{~km}$ ) of the ramp.

The observed magnetic field magnitude profile is broadly consistent with expectations based on theory and earlier observations. The shock structure is self-regulating with the shock electric field adjusting readily to reflect exactly the right number of ions to satisfy the Rankine-Hugoniot conditions. Since for the quasi-perpendicular shock the perpendicular magnetic field component is proportional to the ion density, we also expect the field to be steady. The length of the shock foot (as identified by the extent of the upstream fluctuations) is roughly $150 \mathrm{~km}$. Livesey et al. (1982) found typical shock overshoot (which they defined as being from the beginning of the foot to the bottom of the undershoot) lengths of a few ion inertial lengths, with a mode around 6 . The upstream ion inertial length for the 00:03 shock, on the basis of ACE L1 plasma measurements and the local magnetic field, was $\approx 59 \mathrm{~km}$; from Fig. 4 the overshoot length is $\approx 300 \mathrm{~km}$, consistent with the results of Livesey et al. (1982).

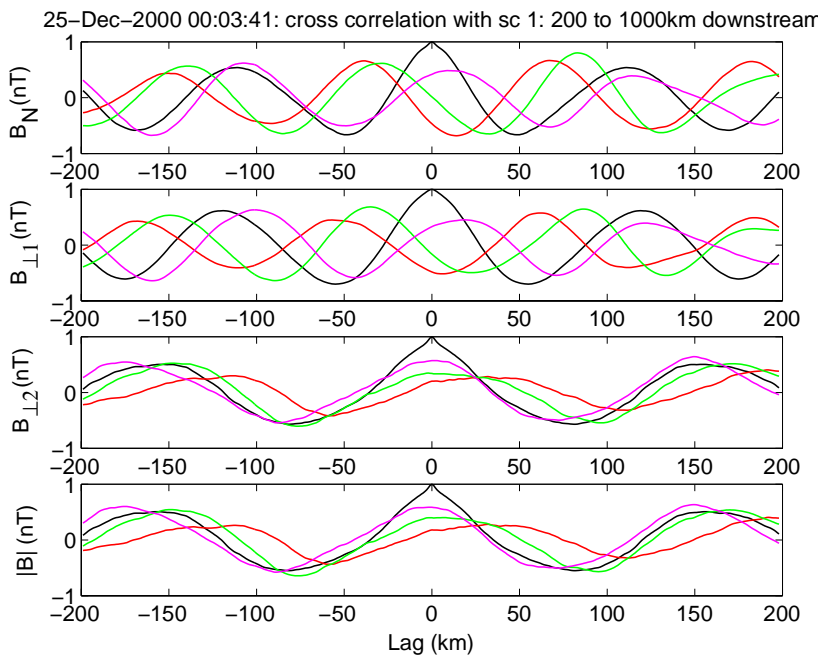

Fig. 5. Cross correlations between spacecraft for data between 200 and $1000 \mathrm{~km}$ downstream of the 00:03 shock. Data for each spacecraft are cross correlated with those from spacecraft 1 for each field coordinate in the same system as Fig. 4. Colours are black, red, green and magenta for spacecraft 1,2, 3 and 4, respectively.

The profile of the two field components perpendicular to the average field direction, $B_{N}$ and $B_{\perp 1}$, is strikingly different to that of the field magnitude. Large amplitude $(\delta B / B \approx$ $0.5)$ non-compressive waves begin after the overshoot, and continue downstream past $1000 \mathrm{~km}$. Unlike the field magnitude variations, the wave activity in the components perpendicular to the field is not well correlated between spacecraft. While statistically similar at all four spacecraft, it is not possible to identify the same structures in more than one time series. This is despite the fact that spacecraft 1, 3 and 4 encountered the shock near-simultaneously (within around $3 \mathrm{~s}$ of each other, similar to the duration of the ramp in the spacecraft frame). These waves are not, therefore, standing relative to the shock surface and are likely to have a scale size less than the inter-spacecraft separation, around $1000 \mathrm{~km}$; since their downstream scale parallel to the shock normal is around $100 \mathrm{~km}$, this is not surprising. This is consistent with the results of Winske and Quest (1988), who found that fluctuations had scales parallel to the shock surface of a few $c / \omega_{\mathrm{i}}$, around $200 \mathrm{~km}$ here.

A wavelet analysis of the wave activity around the shock, not presented here, indicates that the high frequency waves around the shock ramp are right hand polarised relative to the local magnetic field direction in the spacecraft frame. These upstream waves appear to be consistent with upstream whistlers, as predicted at sub-critical shocks (e.g. Mellott and Greenstadt, 1984), although the shocks observed here are supercritcal. The low frequency downstream waves are lefthand polarised relative to the magnetic field, as expected for Alfvén waves, and below the local ion cyclotron frequency. These are consistent with ion cyclotron waves generated by non-gyrotropic proton distributions downstream of the shock, Doppler shifted to lower frequencies by their 


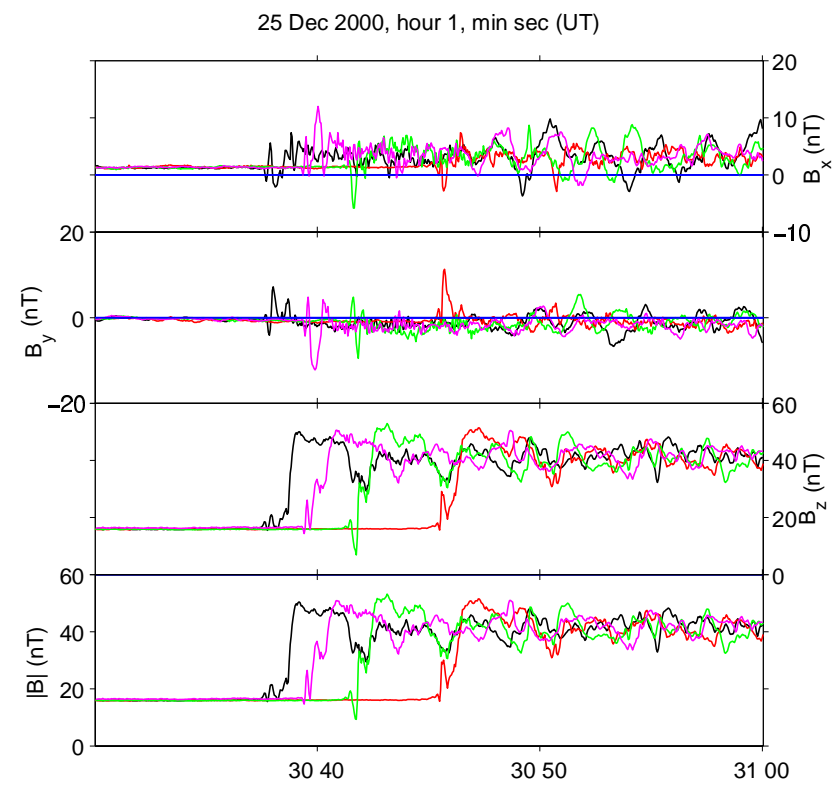

Fig. 6. Four spacecraft observations of a quasi-perpendicular shock. Colours are black, red, green and magenta for spacecraft 1, 2, 3 and 4 respectively. High resolution ( 22 vector/s) GSE data are shown. Note the different ramp profile at each spacecraft.

anti-sunward motion. Wavevectors of these fluctuations have not been estimated although they are approximately circularly polarised, consistent with McKean et al. (1995), who found downstream parallel-propagating ion cyclotron waves in their quasi-perpendicular shock simulations. Analyses of waves downstream of quasi-perpendicular shocks also reveal the presence of mirror modes in addition to Alfvén ion cyclotron waves (Hubert et al., 1989), particularly at frequencies higher than the $\approx 5$ s downstream fluctuations in Fig. 4 (Lacombe et al., 1992). Such modes are likely to be present in the shocks considered here but, without density data, it is not possible unambiguously to identify them.

Cross correlations of magnetic field data, taken between 200 and $1000 \mathrm{~km}$ downstream of the shock, are presented in Fig. 5. To compare data from different spacecraft, the field time series at each spacecraft was first converted to a spatial sample, as for Fig. 4, with $0 \mathrm{~km}$ at the shock ramp. The data were then linearly interpolated onto a regularly spaced grid with $5 \mathrm{~km}$ resolution. For each component, the data at each spacecraft was cross-correlated with that taken at spacecraft 1 . The trace for spacecraft 1 (black), therefore, is the autocorrelation function, peaking at 1 at zero lag. For the two components perpendicular to the magnetic field, the distance between the peaks of the autocorrelation, and hence the scale size of the waves parallel to the shock normal, is around $100 \mathrm{~km}$. The scale size of variations in the field-parallel component and the field magnitude is around $150 \mathrm{~km}$, significantly larger than that of the field-perpendicular variations.

Cross correlations of spacecraft 2, 3 and 4 with spacecraft 1 show that field magnitude variations are generally well correlated (peak correlation around 0.7 ) with a lag near zero, at least for spacecraft 3 and 4, again confirming that these are phase standing structures. Spacecraft 2 peaks at a lag of around $50 \mathrm{~km}$, suggesting that the downstream shock structure may have been slightly different there; spacecraft 2 observed the shock around $30 \mathrm{~s}$ earlier than the other three spacecraft. In the field-perpendicular components, while the peak correlation is good (around 0.8), the correlations do not peak at lags near zero. This could be due to all the spacecraft observing the same waves which would then have to be highly inclined to the shock front. However, since the spacecraft separations along the shock surface were larger than the autocorrelation scale of the observed waves, as discussed above, it is more likely that the four spacecraft simply observed statistically similar waves rather than the same wave fronts. Indeed, examination of the waves in Fig. 4, which are of different amplitudes and have different detailed shapes at different spacecraft, suggests that different spacecraft observed different wave packets.

\section{Non-stationary structures in the shock foot}

It can be seen from Fig. 4 that there was some compressive wave activity in the foot of the 00:03 shock. This is clearer in a later shock, seen at 01:30 at the four spacecraft (Fig. 6). Like the 00:03 event, this shock was nearly perpendicular $\left(\theta_{\mathrm{BN}} \approx 89^{\circ}\right.$ at all four spacecraft) but was travelling outward at around $57 \mathrm{~km} / \mathrm{s}: \mathrm{M}_{\mathrm{A}}$ was around 5.6. While the general shock structure was similar at all four spacecraft, as for the 00:03 shock, there were clear variations in the field magnitude profile at the bottom of the ramp accompanied by fluctuations perpendicular to the mean field. For each of the individual field magnitude profiles, it seems that the shock was rather complex in structure. However, by considering all four profiles, it appears that small scale fluctuations were superimposed on a more uniform shock structure. To examine this effect, the field profiles from the four spacecraft were transformed into a spatial scale relative to the shock normal, in the same manner as for Fig. 4, and then averaged to produce an average shock profile. This is shown in Fig. 7 (left), where the ramp and overshoot are visible, as is the shock foot. The latter is difficult to discern in any of the individual spacecraft data but is clear when they are averaged in this way. Note that the foot ends and the ramp begins at $0 \mathrm{~km}$ on this scale. Field-perpendicular variations are small, as expected, since all the wave activity has been averaged out. This averaged profile was then removed from each of the time series; the residual field variations are shown in Fig. 7 (right). Upstream field fluctuations are visible, but only within the foot; these stopped abruptly at the start of the ramp $(0 \mathrm{~km})$ at all four spacecraft. However, the detailed profile of the fluctuations is different at each spacecraft. This suggests that this highly localised upstream activity is not phase standing but is likely to be continually convected into the shock itself. This is in contrast to the results of Farris et al. (1993), who found phase standing waves upstream of low $\beta$ quasi-perpendicular shocks. The fluctuations seen here may instead be signatures 

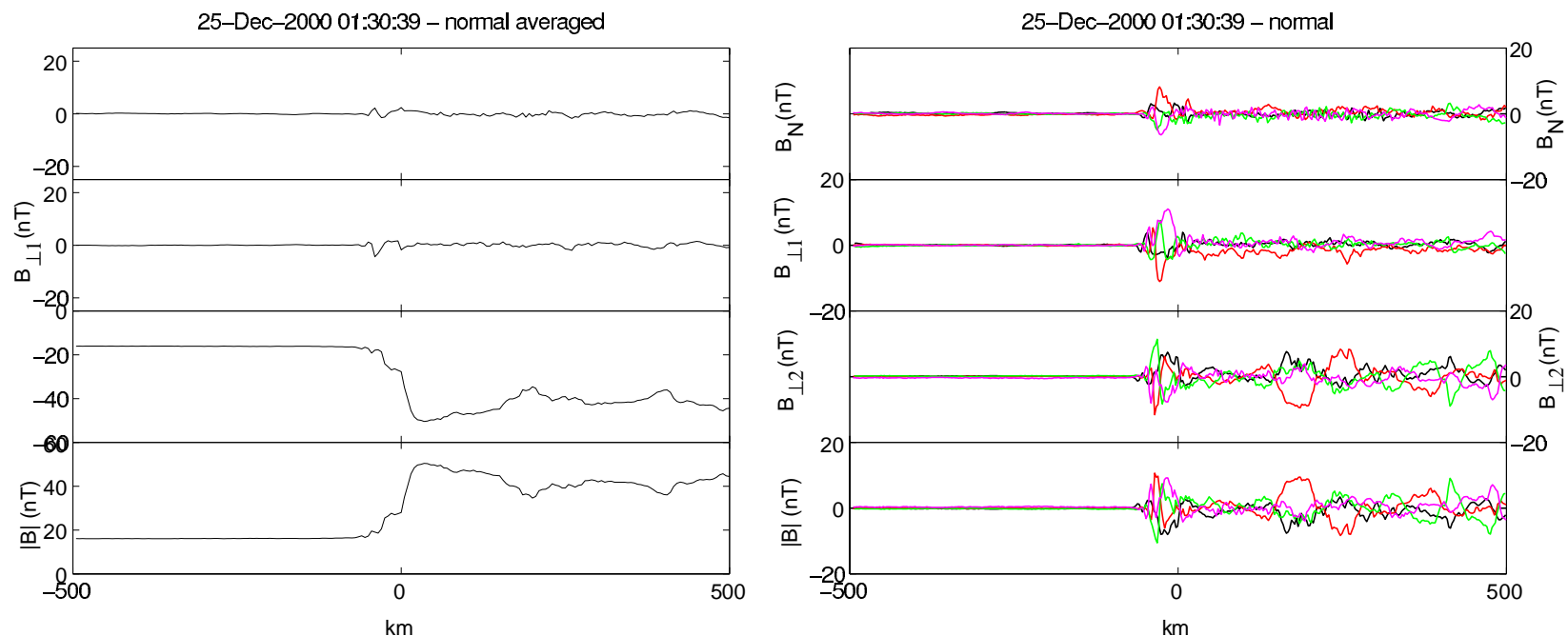

Fig. 7. (left) Magnetic field profile through the shock shown in Fig. 6, averaged over the four Cluster spacecraft. (right) Magnetic field variations, in a normal-aligned coordinate system, after the averaged field profile has been removed. Large waves are visible in the foot and beginning of the ramp.

of unsteady reformation of the nearly-perpendicular supercritical shock (Lembege and Savoini, 1992). The fact that they are different at each spacecraft, although sampled only a few hundred $\mathrm{km}$ and less than $10 \mathrm{~s}$ apart, implies that the spatial and temporal scales of these variations must be small.

The observed fluctuations in the shock foot, which typically last for only one wave period, are generally left-hand polarised relative to the magnetic field in the spacecraft frame and compressive; they are, therefore, consistent with whistler, rather than Alfvén, mode waves. Despite the large peak-to-peak amplitude of these fluctuations (around 20 nT), the shock structure does not appear to be disrupted by them, unlike the case of the quasi-parallel shock (Burgess, 1989b), indicating the stability of the ramp and overshoot when these perturbations are applied. It is also clear, from Fig. 7, that the variations in the foot do not pass through the shock into the downstream region and are not therefore precursors of waves downstream of the shock.

\section{Bowshock propagating through solar wind structures}

The four Cluster spacecraft, by sampling the bowshock at different locations and times, can identify variations in the shock as it passes through solar wind structures. An example of this is presented in Fig. 8, with data taken at around 09:06 on 25 December. The bowshock was travelling Earthwards during this time and spacecraft 2 , being upstream of the others, had passed through it before the time shown in Fig. 8. It was, therefore, sampling solar wind plasma during this time and observed several changes in the solar wind magnetic field direction. The left and right panels show the magnetic field GSE time series for spacecraft 1 and 3 between 09:05:30 and 09:06:30. Superimposed on both (in red) is the data from spacecraft 2, shifted in time such that variations in field an- gle, particularly those in $\theta$, are approximately aligned. The time shift is only 1.75 and 1.27 seconds from spacecraft 2 to 1 and 3 ; the observations of the field angle variations are nearly simultaneous. This procedure of alignment is essentially the same as the normal and velocity estimation method described above and resulted in an estimate of the velocity of these field variations of around $310 \mathrm{~km} / \mathrm{s}$, similar to the ACE solar wind measurements at L1. The general field structure at spacecraft 1 and 3 agree well with that at spacecraft 2, indicating that they observed the same large scale structures. However, both spacecraft 1 and 3 passed through the bowshock during this time and it can be seen from Fig. 8 that the field profiles at the two spacecraft were very different. In particular, spacecraft 3 observed a very wide shock, with small upstream wave activity, while spacecraft 1 passed through a much narrower shock, just before a sharp change in IMF angle, and observed large upstream waves.

It is remarkable that spacecraft 1 and 3 observed shocks that look so different at essentially the same time - the shock ramp at both spacecraft finished around 09:05:05. This inspite of the fact that the spacecraft were separated by around $500 \mathrm{~km}(\delta(X, Y, Z)=(133,379,280) \mathrm{km}$ in GSE $)$. It is clear, therefore, that the bowshock can exhibit a very different character over very small scales, near-simultaneously, when solar wind conditions vary. This reinforces previous simulation results of discontinuities propagating through the bowshock (e.g. Burgess, 1989a), which demonstrated nonequilibrium behaviour and dramatic changes in particle dynamics with considerable upstream injection, in such situations.

The changes in IMF angle in Fig. 8 resulted in changes in $\theta_{\mathrm{BN}}$. Assuming that the normal direction remained constant during this time (probably reasonable, given its stability during the rest of the day) it is possible to estimate $\theta_{\mathrm{BN}}$ from spacecraft 2 data during this interval; this is shown 

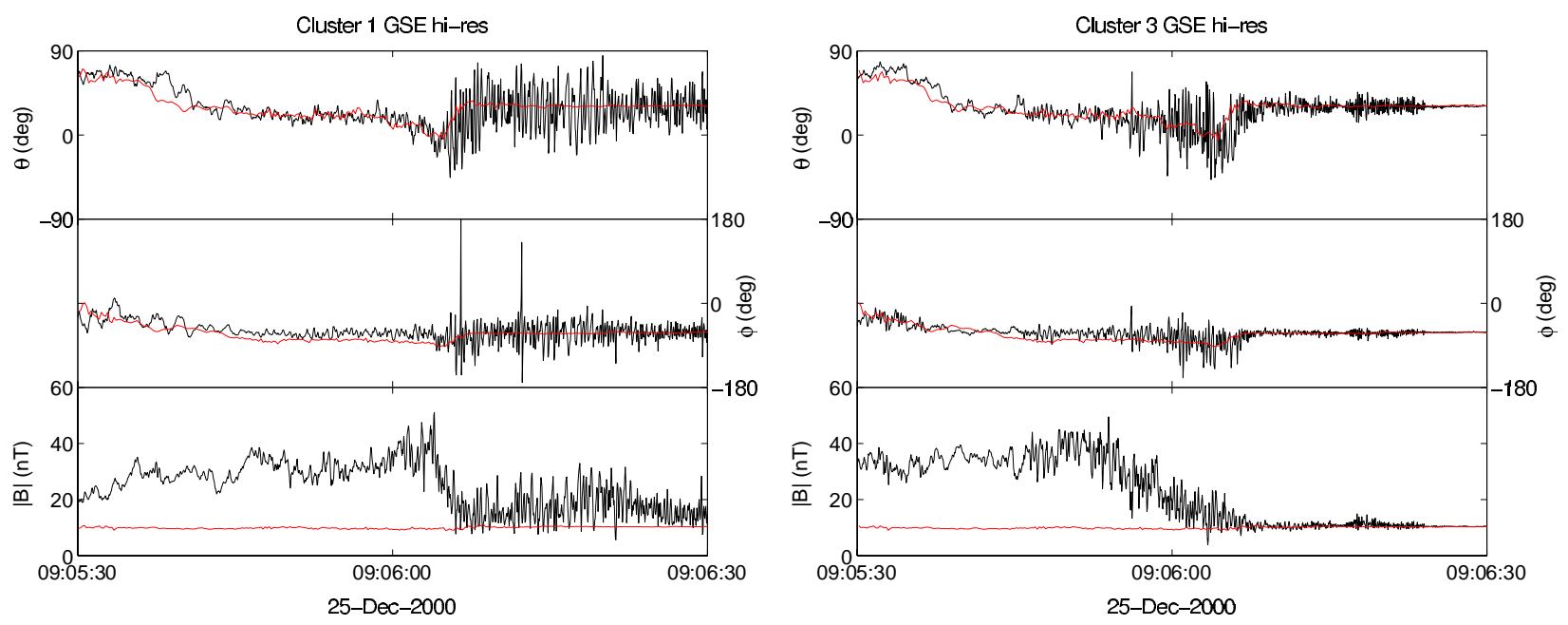

Fig. 8. (left) One minute of high resolution magnetic field data at Cluster 1 (black lines). Spin averaged data from Cluster 2 are superimposed in red, time shifted to align solar wind angle changes. (right) Cluster 3 data in the same format.

in Fig. 9. During the time when spacecraft 3 observed the shock, the field was of rather low inclination and $\theta_{\mathrm{BN}}$ was around $70^{\circ}$, much lower than for most of the day. After the field rotation at 09:06:05, $\theta_{\mathrm{BN}}$ was again near $90^{\circ}$. These variations in the field/normal angle are probably responsible for the different shock profiles, with a wider shock seen at lower $\theta_{\mathrm{BN}}$ at spacecraft 3 and a sharper, nearly perpendicular, shock at spacecraft 1 . However, one would therefore expect lower upstream wave activity at the quasi-perpendicular shock at spacecraft 1 than at 3 while the opposite is the case. It may be that the large upstream wave activity at spacecraft 1 was generated by particles from a nearby, oblique part of the bowshock such as that seen at spacecraft 3 . An analysis of the magnetic connections of the spacecraft to the bowshock, which could corroborate this possibility, will be performed in future work.

\section{Oscillating bowshock}

The four spacecraft observed a highly unusual shock structure between 08:30 and 08:40 on 25 December (Fig. 10). The shock was moving Earthwards, and spacecraft 2 passed into the solar wind before the other three spacecraft, as expected. However, it is clear from Fig. 10 that quasi-periodic oscillations in the field magnitude and direction were present in the magnetosheath at this time. These oscillations, with a typical recurrence time of around $15 \mathrm{~s}$ but only lasting about $5 \mathrm{~s}$, appear to have been carried downstream by the magnetosheath flow - they are visible at spacecraft 2 a few seconds before the other three spacecraft - at around $200 \mathrm{~km} / \mathrm{s}$. Close to the shock itself, the oscillations became more pronounced and spacecraft 2 appears to have experienced large field magnitude variations, which may have been multiple bowshock encounters, before passing into the solar wind around 08:36. The other three spacecraft entered the solar wind a few minutes later and all four spacecraft then encountered similar quasi-periodic magnetic field variations upstream for around two minutes before the IMF became settled.

The cause of these oscillations is not clear. The IMF $B_{Z}$ was rather small at this time, so $\theta_{\mathrm{BN}}$ may have been low. Estimating a timing-derived normal, using the last bowshock crossing for each spacecraft, leads to the data shown in Table 1 for this shock, with a significantly different normal to the other shocks on this day. However, the timing method should be treated with caution in this case, since the bowshock was clearly highly variable at this time. On the basis of the derived normal, $\theta_{\mathrm{BN}}$ was around $60^{\circ}$, although this value is rather uncertain because of the uncertainties in normal estimation. The shock, however, was probably oblique but not quasi-parallel - the final shock transition at each spacecraft was rather sharp.

Figure 10 shows that the magnetosheath near the bowshock, and perhaps the bowshock itself, contained large amplitude quasi-periodic oscillations at this time. These oscillations were carried Earthwards with the sheath plasma; they were not, therefore, standing structures which were part of the bowshock, such as the overshoot. It is not clear whether the field magnitude variations seen by spacecraft 2 , before its bowshock crossing and entry into the solar wind, were also bowshock crossings or the same oscillations as seen in the sheath. The fact that oscillations of a similar period were visible in the IMF upstream of the shock might suggest that these were of solar wind origin but the fact that they died away upstream of the shock suggests that they were generated by shock processes such as ions propagating upstream. However, unlike the upstream waves in Fig. 6, the upstream variations in Fig. 10 are coherent between the spacecraft, implying that they have a large $(>500 \mathrm{~km})$ length scale perpendicular to the shock normal. At this time, the origin of these quasi-periodic structures is not clear - they will be investigated further in a later paper. 


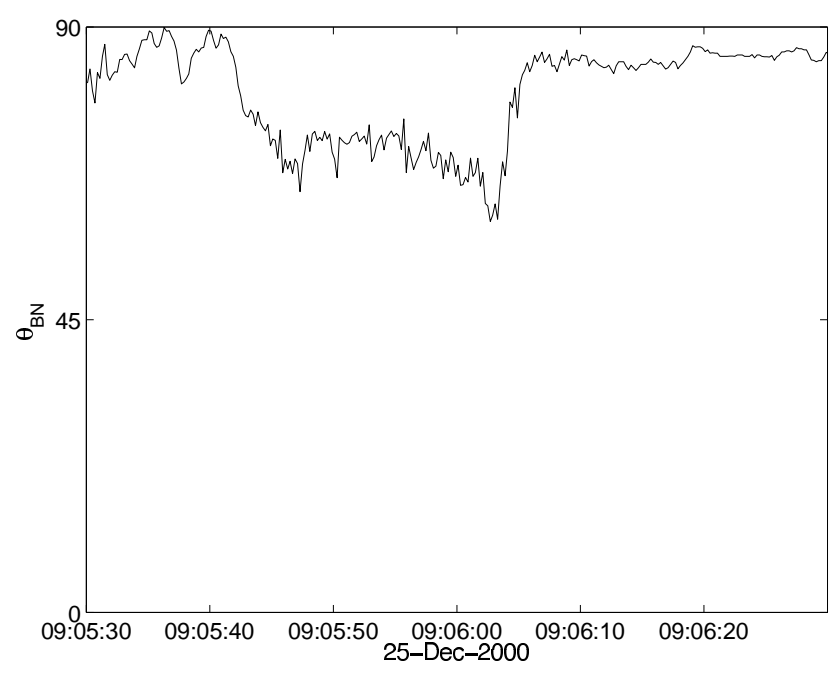

Fig. 9. $\theta_{\mathrm{BN}}$ values at Cluster 2, calculated on the basis of local magnetic field data and a normal similar to that at recent shocks, for the same time period as Fig. 8.

\section{Bowshock motion within the formation}

Despite the fact that spacecraft 2 was less than $1000 \mathrm{~km}$ upstream of the other three spacecraft during 25 December, there were a number of occasions when the bowshock reversed direction within the formation tetrahedron. One example is shown in Fig. 11. Here, spacecraft 2 remained in the solar wind throughout the interval. The other three spacecraft started in the solar wind and were passed by the bowshock moving sunwards; this reversed and moved back over 1,3 and 4 without reaching spacecraft 2 . Surprisingly, there are no obvious magnetic field features in the spacecraft 2 time series to explain the reversal of the bowshock motion. The propagation time of solar wind features between the spacecraft was only a few seconds (as can be seen for the small increase in $B_{Y}$ near the end of the interval) so, if any change in IMF conditions caused the bowshock motion, it should be visible in Fig. 11. It is possible that one or more plasma parameters (velocity, density, temperature) may have changed during this time, but such changes are usually accompanied by at least small simultaneous IMF changes. Without accompanying plasma data it is difficult to determine the causes of this bowshock motion but it may reflect inherent instability of the shock surface. We note that the timing differences of the passage of spacecraft 1, 3 and 4 through the two bowshock crossings were different, suggesting that the shock was at a slightly different orientation and moving at a different velocity, in the two cases. It is not possible to determine a normal from the relative timings of the three spacecraft, however.

While Fig. 11 shows a period when the bowshock passed over three of the spacecraft while one remained in the solar wind, Fig. 12 shows the opposite case, where three remained in the magnetosheath while one repeatedly passed through the bowshock. The bowshock remained sunwards of the formation for most of this period but moved Earthwards over

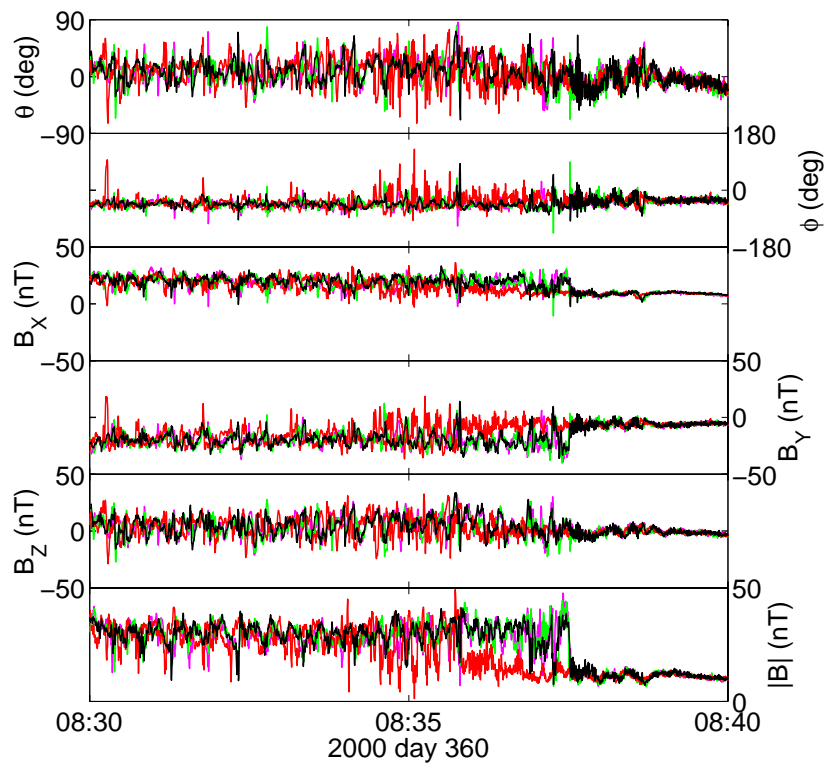

Fig. 10. Four spacecraft magnetic field data showing quasi-periodic oscillations in the magnetosheath and around the bowshock, in the same format as Fig. 6.

spacecraft 2 and returned sunwards several times. Some of these crossings are extremely slow, lasting at least two minutes, implying a bowshock velocity of under $1 \mathrm{~km} / \mathrm{s}$. Indeed, between 6:55 and 7:00, there were some partial crossings where the shock appears to have been moving over the spacecraft but reversed before completely passing over it. Between $7: 13$ and 7:18, while spacecraft 2 was in the solar wind, the other three spacecraft observed significant changes in magnetosheath field. It may be that the bowshock came close to the spacecraft during this time. Later work will examine these and other crossings in detail. Such events offer the prospect of determining both solar wind causes for rapid bowshock acceleration and magnetosheath responses to such motion.

\section{Discussion}

A first examination of Cluster, multi-spacecraft magnetic field measurements of the quasi-perpendicular low $\beta(\beta \approx$ $0.1)$, moderate Mach number $\left(\mathrm{M}_{\mathrm{A}} \approx 5\right)$ bowshock, has revealed a wide range of shock phenomena in this unusual regime:

A remarkably stable bowshock normal, even while the shock is in rapid motion, and good agreement of the timingderived normal with a model (Peredo et al., 1995);

A mean deviation of the coplanarity vector to the timingderived normal of around $30^{\circ}$, for a range of up and downstream averaging intervals;

Phase-standing field magnitude variations downstream of the shock, with a scale of around $150 \mathrm{~km}$ (about $2 c / \omega_{\mathrm{i}}$ ) parallel to the shock normal; 


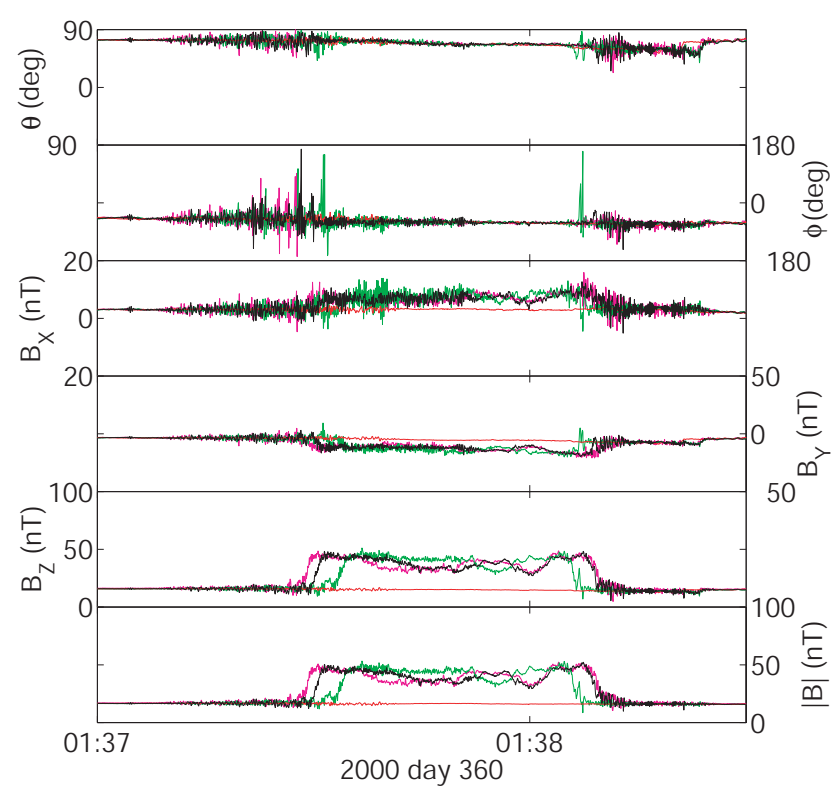

Fig. 11. Ninety seconds of magnetic field data at all four spacecraft. Traces for each spacecraft are shown in a different colour: black, red, green and magenta for spacecraft $1,2,3$ and 4, respectively. Spacecraft 1, 3 and 4 briefly entered the magnetosheath during this time.

Downstream non-standing ion cyclotron waves, with a smaller scale than the magnitude variations and a scale perpendicular to the shock normal considerably less than $1000 \mathrm{~km}$;

Compressive (probably whistler) fluctuations of significant amplitude in the foot of one shock, which are not phase standing and not present in the ramp;

Variations in bowshock properties with solar wind conditions, on the scale of seconds;

Quasi-periodic oscillations up and downstream of a highly variable shock;

Rapid bowshock acceleration within the formation tetrahedron.

A number of the above results confirm earlier single or dual spacecraft studies or simulations. Some, however, are new. This paper has only considered one day of data and one shock regime, the quasi-perpendicular low $\beta$ shock. Future work will address other regimes as well as following up the open questions left by this paper. The Cluster data set is already a rich and varied one, with a large "zoo" of shock phenomena available for study. More detailed analysis, and in particular combination of data from field and particle instruments, will greatly improve understanding of collisionless shock phenomena.

Acknowledgements. The authors are grateful to J. A. Slavin (GSFC) for his assistance in the calculation of model normals, the ACE SWEPAM team for their rapid supply of ACE SWEPAM data and both referees for their helpful comments. Cluster analysis at Imperial College is supported by PPARC(UK); T. Horbury is supported by a PPARC Fellowship.

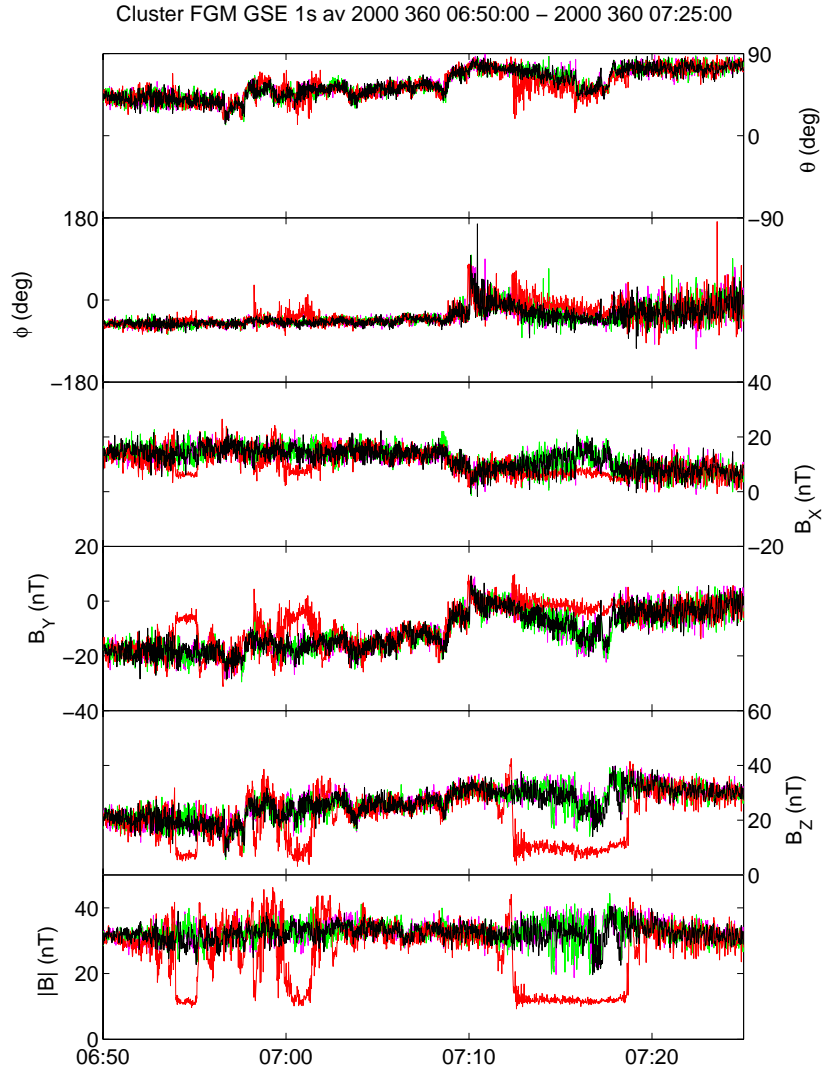

Fig. 12. Cluster magnetic field data during a time when the bowshock passed repeatedly over spacecraft 2 . Traces for each spacecraft are shown in a different colour: black, red, green and magenta for spacecraft 1,2, 3 and 4, respectively.

Topical Editor G. Chanteur thanks C. Lacombe and another referee for their help in evaluating this paper.

\section{References}

Balogh, A., Carr, C. M., Acuña, M. H., Dunlop, M. W., Beek, T. J., Brown, P., Fornacon, F.-H., Georgescu, E., Glassmeier, K. H., Harris, J., Musmann, G., Oddy, T., and Schwingenschuh, K.: The Cluster magnetic field investiagation: overview of in-flight performance and initial results, Ann. Geophysicae, this issue, 2001.

Burgess, D.: On the effect of a tangential discontinuity on ions specularly reflected at an oblique shock, J. Geophys. Res., 94, 472, 1989a.

Burgess, D.: Cyclical behaviour at quasi-parallel collisionless shocks, Geophys. Res. Lett., 16, 345, 1989b.

Dunlop, M. W. and Woodward, T. I.: Multi-spacecraft discontinuity analysis: orientation and motion, in Analysis methods for multispacecraft data, (Eds) Paschmann, G. and Daly, P. W., International Space Science Institute, Bern, 1998.

Dunlop, M. W., Balogh, A., Glassmeier, K.-H., et al.: Four-point application of magnetic field analysis tools: the curlometer and discontinuity analyser, Ann. Geophysicae, this issue, 2001.

Farris, M. H., Russell, C. T., and Thomsen, M. F.: Magnetic structure of the low beta, quasi-perpendicular shock, J. Geophys. Res., 98, 15 285-15 294, 1993. 
Goodrich, C. C.: Numerical simulations of quasi-perpendicular collisionless shocks, in: Collisionless shocks in the heliosphere: reviews of current research, (Eds) Tsurutani, B. T. and Stone, R. G., AGU, Washington, D. C., 1985.

Hubert, D., Perche, C., Harvey, C. C., Lacombe, C., and Russell, C. T.: Observation of mirror waves downstream of a quasiperpendicular shock, Geophys. Res. Lett., 16, 159-162, 1989.

Krauss-Varban, D.: Waves associated with quasi-parallel shocks: generation, mode conversion and implications, Adv. Space Res., 15 8/9, 271, 1995.

Lacombe, C., Pantellini, F. G. E., Hubert, D., Harvey, C. C., Mangeney, A., Belmont, G., and Russell, C. T.: Mirror and Alfvénic waves observed by ISEE 1-2 during crossings of the Earth's bow shock, Ann. Geophysicae, 10, 772-784, 1992.

Lembege, B. and Savoini, P.: Nonstationarity of a two-dimensional quasiperpendicular superciritcal collisionless shock by selfreformation, Phys. Fluids B, 4, 11, 3533-3548, 1992

Leroy, M. M., Winske, D. Goodrich, C. C., Wu, C. W., and Papadopoulos, K.: The structure of perpendicular bow shocks, J. Geophys. Res., 87, 5081, 1982.

Livesey, W. A., Kennel, C. F., and Russell, C. T.: ISEE-1 and 2 observations of magnetic field strength overshoots in quasiperpendicular bow shocks, Geophys. Res. Lett., 9, 1037-1040, 1982.

McKean, M. E., Omidi, N., and Krauss-Varban, D.: Wave and ion evolution downstream of quasi-perpendicular bow shocks, J. Geophys. Res., 100, 3427-3437, 1995.

Mellott, M. M. and Greenstadt, E. W.: The structure of oblique subcritical bow shocks: ISEE 1 and 2 observations, J. Geophys. Res., 89, 2151, 1984.

Peredo, M., Slavin, J. A., Mazur, E., and Curtis, S. A.: 3- dimensional position and shape of the bow shock and their variation with Alfvénic, sonic and magnetosonic Mach numbers and interplanetary magnetic-field orientation, J. Geophys. Res., 100, 7907-7916, 1995.

Quest, K. B.: Simulations of high Mach number perpendicular shocks with resistive electrons, J. Geophys. Res., 91, 8805, 1986.

Russell, C. T. and Farris, M. H.: Ultra low frequency waves at the Earth's bow shock, Adv. Space Res., 15, 8/9, 285-296, 1995.

Schwartz, S. J., Thomsen, M. F., and Gosling, J. T.: Ions upstream of the Earth's bow shock: a theoretical comparison of alternative source populations, J. Geophys. Res., 88, 2039-2047, 1983.

Sckopke, N., Paschmann G., Bame, S. J., Gosling, J. T., and Russell, C. T.: Evolution of ion distributions across the nearly perpendicular bow shock: specularly and nonspecularly refecteing ions, J. Geophys. Res., 88, 6121, 1983.

Scudder, J. D., Mangeney, A., Lacombe, C., Harvery, C. C., Aggson, T. L., Anderson, R. R., Gosling, J. T., Paschmann, G., and Russell, C. T.: The resolved layer of a collisionless, high $\beta$, supercritical, quasi-perpendicular shock wave 1 . RankineHugoniot geometry, currents, and stationarity, J. Geophys. Res., 91, 11 019-11 052, 1986.

Song, P. and Russell, C. T.: Theory of multipoint data analysis, in ESA SP-449 Proc. Cluster-II workshop on multiscale/multipoint plasma measurements, (Ed) Harris, R. A., ESA Publications division, Noordwijk, 2000.

Thomas, V. A.: Dimensionality effects in hybrid simulations of high Mach number collisionless perpendicular shocks, J. Geophys. Res., 94, 12 009-12 014, 1989.

Winske, D. and Quest, K. B.: Magnetic field and density fluctuations at perpendicular supercritical collisionless shocks, J. Geophys. Res., 93, 9681-9693, 1988. 